\title{
THE WORD PROBLEM AND RELATED RESULTS FOR GRAPH PRODUCT GROUPS
}

\author{
K. J. HORADAM
}

\begin{abstract}
A graph product is the fundamental group of a graph of groups. Amongst the simplest examples are HNN groups and free products with amalgamation.

The torsion and conjugacy theorems are proved for any group presented as a graph product. The graphs over which some graph product has a solvable word problem are characterised. Conditions are then given for the solvability of the word and order problems and also for the extended word problem for cyclic subgroups of any graph product. These results generalise the known ones for HNN groups and free products with amalgamation.
\end{abstract}

1. Introduction. Interest in groups which are graph products (that is, fundamental groups of graphs of groups in the terminology of Bass and Serre) has been marked in recent years, in view of their utility in combinatorial group theory. The normal form theorem (Britton's Lemma) and the conjugacy theorem (Collins' Lemma) for the simplest graph products-the HNN groups and free products with amalgamation-have been vital in the study of decision problems for these groups.

This paper proves the torsion and conjugacy theorems for graph products. Decision problems for a graph product cannot be considered without first paying attention to the associated graph. The graphs over which some graph product has a solvable word problem are completely characterised. (Any such solution is not presentation-free: a group may have two presentations as a graph product over distinct graphs, of which only one has a solvable word problem.)

Conditions are given for the solvability of the word and order problems, and also for the solvability of the extended word problem for cyclic subgroups of graph products. The known results for HNN groups, free products with amalgamation and their various generalisations follow immediately as particular cases. A class of graph products is defined for which the word problem and extended word problem for infinite cyclic subgroups are both solvable.

The work is couched in terms of (Brandt) groupoids, which provide a most natural setting for proofs of this kind. Hence a graph product is considered as the loop group of a connected mapping cylinder of a diagram of groups, in which all the group homomorphisms are monic. The reader is referred to [3] for the basic constructions of the theory of groupoids, to [8] for the Bass-Serre theory, and to [6, Chapter V] for the theory of computability.

Received by the editors May 28, 1980.

1980 Mathematics Subject Classification. Primary 20F10; Secondary 20L10.

Key words and phrases. Groupoid, graph of groups, fundamental group, word problem, extended word problem, torsion theorem, conjugacy theorem. 
My thanks go to Dr. R. Bieri for first interesting me in the word problem, to Melbourne University for its hospitality during the preparation of this paper, and especially to Professor C. F. Miller III for his very helpful comments.

2. The normal form for graph products. Results in this section rely heavily on the Spelling Lemma [9], which generalises Britton's Lemma, and follow the terminology there. Exposition is purposely patterned on that of Lyndon and Schupp [5, IV.2] for HNN extensions and free products with amalgamation. Throughout what follows, $D=(E, V)$ is a connected directed graph with edge set $E$, vertex set $V$ and left and right vertex maps $\lambda, \rho: E \rightarrow V ;(D, A)$ is a group diagram with monic edge homomorphisms, and $G$ is the mapping cylinder groupoid of $(D, A)$ under the map $m:(D, A) \rightarrow m(D, A)=G$. That is to say, $(D, A)$ consists of a group $A_{v}$ (with identity $\left.1_{v}\right)$ for each $v$ in $V$, and a set of group monomorphisms $\left\{A_{e}: A_{\lambda_{e}} \rightarrow A_{\rho e}, e\right.$ $\in E\}$. If $A_{v}$ has a presentation $\left\langle X_{v} \mid R_{v}\right\rangle$ for each $v$ in $V$, the groupoid $G$ has a presentation as follows:

identities: Id $G=\left\{1_{v}: v \in V\right\}$;

generators: $X=\left(\cup_{v \in V} X_{v}\right) \cup\left\{t_{e}: e \in E\right\}$;

relations: $R=\left(\cup_{v \in V} R_{v}\right) \cup\left\{t_{e}^{-1} x t_{e}=A_{e}(x), e \in E, x \in X_{\lambda_{e}}\right\}$;

identity maps on generators: $\lambda x=1_{v}, x \in X_{v} ; \lambda t_{e}=1_{\lambda_{e}}$;

$$
\rho x=1_{v}, x \in X_{v} ; \rho t_{e}=1_{\rho e} \text {. }
$$

Note that context alone determines whether the symbols $\lambda$ and $\rho$ refer to left and right identity maps on the groupoid $G$ or left and right vertex maps on the graph $D$.

Let $G_{0}$ be the group of loops of $G$ at a distinguished identity $1_{0}$ in Id $G$, so that $G_{0}$ is a graph product. (Alternatively, the graph product may be obtained as a quotient of $G$ by contracting a maximal acyclic subgroupoid to $1_{0}$.) For example, the graph products corresponding to group diagrams

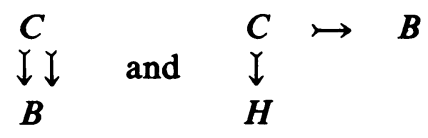

are the HNN extension with base $B$ and associated subgroup $C$, and the free product with amalgamation $H{ }_{c} B$, respectively. In comparison, the Bass-Serre theory would represent the latter as $H \stackrel{C}{ } B$; an edge $C \hookrightarrow B$ in a group diagram could be given as $C \stackrel{C}{C}$ using graphs of groups.

Every element of $G$ may be represented as an element of the set $S^{*}$ of sequences of the form

$$
a_{0}, s_{1}, \ldots, s_{n}, a_{n} \quad(n>0)
$$

such that

(i) each $a_{i}$ is a member of a vertex group $A_{v_{i}}$,

(ii) each $s_{i}$ is an edge-symbol $t_{e_{i}}$ or its inverse, and

(iii) $\rho a_{i}=\lambda s_{i+1}, 0<i<n-1$ and $\rho s_{i}=\lambda a_{i}, 1<i<n$.

Every element of $G_{0}$ may be represented as an element of $S^{*}$ for which the further condition

(iv) $\lambda a_{0}=\rho a_{n}=1_{0}$, holds. 
Definition 2.1. A sequence $\sigma=a_{0}, s_{1}, \ldots, s_{n}, a_{n},(n>0)$ is said to be reduced (or irreducible) if there is no consecutive subsequence of form either

(i) $s_{i}=t_{e}^{-1}, a_{i} \in A_{\lambda e}, s_{i+1}=t_{e}$ for some edge $e$ or

(ii) $s_{i}=t_{e}, a_{i} \in A_{e}\left(A_{\lambda e}\right), s_{i+1}=t_{e}^{-1}$ for some edge $e$.

If $\sigma$ is reduced, its reduced length $\|\sigma\|$ is defined to be $\|\sigma\|=n$.

If $\sigma$ is not reduced, a subsequence of form (i) may be replaced by $A_{e}\left(a_{i}\right)$ and a subsequence of form (ii) by the unique element $a$ in $A_{\lambda e}$ for which $A_{e}(a)=a_{i}$, to give a new sequence equivalent to $\sigma$. The product of elements in distinct reduced sequences of $S^{*}$ may be equal in $G$, in view of the quasi-commuting relations

$$
t_{e} A_{e}(a)=a t_{e}
$$

and

$$
A_{e}(a) t_{e}^{-1}=t_{e}^{-1} a, \text { for } e \text { in } E \text { and } a \text { in } A_{\lambda e} .
$$

However, each reduced sequence may be "left-justified" to take account of this, giving a normal form for each element in $G$. This is done by selecting, for each edge $e$ in $D$, a right transversal $T_{e}$ of $A_{e}\left(A_{\lambda_{e}}\right)$ in $A_{\rho e}$, with the assumption that $1_{\rho e}$ is the representative of $A_{e}\left(A_{\lambda e}\right)$.

Definition 2.2. A normal form (relative to $\left\{T_{e}: e \in E\right\}$ ) in $S^{*}$ is a sequence $\sigma=a_{0}, s_{1}, \ldots, a_{n},(n \geqslant 0)$ such that

(i) if $s_{i}=t_{e}$ then $a_{i}$ is a coset representative of $A_{e}\left(A_{\lambda_{e}}\right)$ in $A_{\rho e}$,

(ii) if $s_{i}=t_{e}^{-1}$ then $a_{i}=1_{\lambda e}$, and

(iii) there is no consecutive subsequence $t_{e}^{-1}, 1_{\lambda e}, t_{e}$ or $t_{e}, 1_{\rho e}, t_{e}^{-1}$.

These definitions combine with the Spelling Lemma and the embedding theorem for graph products [1, Theorem 6.2] to produce the expected normal form theorem in its two equivalent statements.

TheORem 2.3. (The Normal Form Theorem for Mapping Cylinder Groupoids.) (I) (Smythe [1], [9]). The group $A_{v}$ is embedded in $G$ by the map $m_{v}: a \rightarrow a$ for every $v$ in $D$. If $a$ word in $G$ is represented by two reduced sequences $\sigma$ and $\tau$ then $\|\sigma\|=\|\tau\|$. In particular, if $\sigma=a_{0}, s_{1}, \ldots, a_{n}$ and $n \geqslant 1$ and the product $a_{0} s_{1} \ldots a_{n} \in m_{v}\left(A_{v}\right)$ for some $v$, then $\sigma$ is not reduced.

(II) Every element $g$ of $G$ has a unique representation as $g=a_{0} s_{1} \ldots a_{n}$ where $a_{0}, s_{1}, \ldots, a_{n}$ is a normal form.

That (I) and (II) are equivalent, follows without difficulty. Restriction throughout to elements of $G_{0}$ gives The Normal Form Theorem for Graph Products. It is equally easy to produce a "right-justified" normal form which, after accounting for the slightly different graphs involved, may be shown equivalent to that given by Higgins $[2, \S 3]$ using the Bass-Serre theory.

Definition 2.4. A loop $g=a_{0} s_{1} \ldots a_{n-1} s_{n}$ of $G$ is cyclically reduced, if all cyclic permutations of the sequence $a_{0}, s_{1}, \ldots, a_{n-1}, s_{n}$ are reduced.

Every loop of $G$ is conjugate to a cyclically reduced loop.

The torsion and conjugacy theorems for graph products follow from (2.3), using the obvious modifications of the corresponding proofs for HNN extensions and free products with amalgamation [5, IV.2]. 
THEOREM 2.5 (Torsion). Every loop of finite order in $G$ is a conjugate of an element of finite order in a terminal group $A_{p e}$ for some $e$ in $D$. Thus $G_{0}$ has elements of finite order $\boldsymbol{n}$ if and only if a terminal group has elements of finite order $\boldsymbol{n}$.

(Note that if $a \in A_{\lambda e}$ and $a^{n}=1_{\lambda e}$ then $\left(A_{e}(a)\right)^{n}=1_{\rho e}$ in $A_{\rho e}$ )

THEOREM 2.6 (Conjugacy). Let $g=a_{0} s_{1} \ldots s_{n},(n>1)$, and $h$ be conjugate cyclically reduced loops of $G$. Then $\|g\|=\|h\|$, and $g$ can be obtained from $h$ by taking a suitable cyclic permutation of $h$, ending in $s_{n}$, and then conjugating by an element $z$, where $z \in A_{\lambda e}$ if $s_{n}=t_{e}^{-1}$, and $z \in A_{e}\left(A_{\lambda_{e}}\right)$ if $s_{n}=t_{e}$.

3. The word problem for graph products. Scrutiny is first turned to the word problem for any (countable) groupoid. For a presentation of groupoid $\Gamma$, this is the problem of discovering whether or not there is an algorithm to determine if an arbitrary element of $\Gamma$ lies in Id $\Gamma$. It splits into two subproblems.

QUESTION 3.1. Is there an algorithm to determine whether or not an arbitrary element of $\Gamma$ is a loop?

QUESTION 3.2. Is there an algorithm to determine whether or not an arbitrary loop of $\Gamma$ is trivial?

The latter question translates the word problem for groups into groupoid terms, but the former question seeks knowledge of the general structure of $\Gamma$ as a groupoid. This prompts the following definition.

DEFINITION 3.3. A groupoid $\Gamma$ is recursively presented if it has a presentation $(\Gamma=\langle X \mid R\rangle$; Id $\Gamma ; \lambda, \rho: X \rightarrow \operatorname{Id} \Gamma)$ for which

(i) the set of generators $X$ is recursively enumerable (r.e.);

(ii) the set of relators $R$ is r.e.;

(iii) the set of identities Id $\Gamma$ is recursive;

(iv) the identity maps $\lambda, \rho: X \rightarrow \operatorname{Id} \Gamma$ are partially recursive.

Question 3.1 may be rephrased as

Question $3.1^{\prime}$. Are the left and right identity maps $\lambda, \rho: \Gamma \rightarrow \operatorname{Id} \Gamma$ (with domain $\Gamma)$ partially recursive?

This problem makes sense when 3.3(i)-(iii) hold, and is then answerable in the affirmative if and only if 3.3(iv) is true.

In the case that $\Gamma$ is a mapping cylinder $G=m(D, A)$, necessary and sufficient conditons may be given to ensure that $\Gamma$ is recursively presented.

Definition 3.4. (a) The graph $D$ is recursive if

(i) $E$ and $V$ are recursive sets, and

(ii) the vertex maps $\lambda, \rho: E \rightarrow V$ are partially recursive (with domain $E$ ).

(b) The group diagram $(D, A)$ is recursively presented if

(i) $D$ is recursive,

(ii) $A_{v}$ is a recursively presented group for each $v$ in $V$ and the presentations are uniformly given from $V$.

(iii) $A_{e}$ is partially recursive for each $e$ in $E$ and uniformly given from $E$.

With this definition in mind, advantage may be taken of the set isomorphisms Id $G \cong V$ and $E^{*}=\left\{t_{e}: e \in E\right\} \cong E$, to answer Question 3.1 for graph products. 
TheOREM 3.5. The groupoid $G=m(D, A)$ is recursively presented and $E^{*}$ is recursive if and only if $(D, A)$ is recursively presented.

Proof relies on the facts that the identity maps may be built up effectively from the vertex maps and conversely that the restricted identity maps determine the vertex maps.

Thus the directed graphs for which some graph product has a solvable word problem are precisely those with recursive edge and vertex sets and partially recursive vertex maps. (For example, such a graph with trivial groups at the vertices will have a graph product with a solvable word problem.) Any finite connected directed graph satisfies these conditions; for consistency the trivial graph $D_{0}$ with one vertex and no edges is considered to do so.

In practical terms the requirement of partial recursiveness of vertex maps can usually be satisfied by judicious labelling of the edges and vertices. However, graphs whose vertex maps are not partially recursive certainly exist: if $f: Z^{+} \rightarrow Z^{+}$ is a nonpartially recursive map on the positive integers then the directed graph $D_{f}$ with edges labelled by $\operatorname{dom}(f)$, initial vertex given by $\lambda(n)=1$ and terminal vertices given by $\rho(n)=f(n)$ for all $n$ in $\operatorname{dom}(f)$, is such a one. It follows, for instance, that the particular presentation of the trivial group as the graph product of the graph $D_{f}$, with trivial groups (presented as such) at the vertices, will not have a solvable word problem.

It is now possible to give sufficient conditions for a solution of the word problem for graph products. Clearly by $2.3(\mathrm{I})$ the condition 3.6(i) is also necessary. Hereinafter, we assume that $G$ is (presented as) the mapping cylinder of a recursively presented group diagram.

Theorem 3.6 (Word Problem). Suppose the following conditions hold.

(i) $A_{v}$ has a uniformly solvable word problem for every $v$ in $D$.

(ii) $A_{e}\left(A_{\lambda e}\right)$ has a uniformly solvable extended word problem in $A_{\rho e}$ for every $e$ in $D$.

Then $G$ has a solvable word problem, and consequently so has $G_{0}$.

Proof. There is an algorithm to decide whether a given word of $G$ is a loop or not; if not, it cannot be an identity. If $g$ is a loop in $G$ represented by a sequence $\sigma$ then $\sigma$ may be reduced to an irreducible sequence $\sigma^{*}$ whose product in $G$ equals $g$. The reduction process is algorithmic, as it can be told which words of $A_{\rho e}$ represent elements of $A_{e}\left(A_{\lambda_{e}}\right)$, and $A_{e}(a)$ can be calculated from $a$ and vice versa. If $\left\|\sigma^{*}\right\|>1$ then $g$ is not an identity by 2.3. If $\left\|\sigma^{*}\right\|=0$ then $g$ equals an element in a (known) vertex group $A_{v}$, and an algorithm decides whether or not $g$ equals $1_{v}$.

The corresponding results for free products with amalgamation and HNN extensions follow immediately.

COROLlARY 3.7. Under the conditions of 3.6 the extended word problem for $A_{v}$ in $G$ (and for its conjugates in $G_{0}$ ) is solvable for every $v$ in $D$.

COROllary 3.8 (Order). Suppose G satisfies the conditions of 3.6. If the order problem is uniformly solvable for $A_{v}$ for every $v$ in $D$, then the order problem is solvable for $G$, and hence for $G_{0}$. 
Proof. Let $h$ be any loop of $G$. The process of finding words $z$ and $h^{*}$ such that $h=z^{-1} h^{*} z$ and $h^{*}$ is cyclically reduced, is algorithmic under the conditions of 3.6 since $h$ has finite reduced length. If $\left\|h^{*}\right\| \geqslant 1$ then by $2.6, h$ is not conjugate to any element of a vertex group and thus has infinite order. If $\left\|h^{*}\right\|=0$ then $h^{*} \in A_{v}$ for some (known) $v$ in $D$, and $h$ has finite order if and only if $h^{*}$ has finite order. If the order of $h$ is finite, it can be found because the word problem for $A_{v}$ is solvable.

4. The extended word problem. The extended word problem for cyclic subgroups of graph products is investigated.

THEOREM 4.1. Suppose $G$ satisfies the conditions of 3.6. If the extended word problem for infinite cyclic subgroups of $A_{v}$ is uniformly solvable for every $v$ in $D$, then the extended word problem for infinite cyclic subgroups of $G$ (and hence $G_{0}$ ) is solvable.

Proof. Let $f$ be a (nontrivial) loop of $G$ which is known to generate an infinite cyclic subgroup $F$ of $G$. Let $g$ be any word of $G$ : if $g$ is not a loop at $1_{\lambda f}$, then $g \notin F$. Determine algorithmically, words $x$ and $y$ and cyclically reduced words $f^{*}$ and $g^{*}$ such that $f=x^{-1} f^{*} x$ and $g=y^{-1} g^{*} y$. If $\left\|f^{*}\right\|>1$ and $\left\|g^{*}\right\|$ is either zero or not divisible by $\left\|f^{*}\right\|$, then $g \notin F$. If $\left\|f^{*}\right\|$ and $\left\|g^{*}\right\|$ are both positive, and $k$ is a positive integer such that $\left\|g^{*}\right\|=k\left\|f^{*}\right\|$ then $g \in F$ if and only if $g=f^{ \pm k}$. Because $G$ has a solvable word problem this criterion is decidable algorithmically. If $\left\|f^{*}\right\|=0$ but $\left\|g^{*}\right\|>0$ then $g \notin F$. Finally, if $\left\|f^{*}\right\|=\left\|g^{*}\right\|=0$, so that $f^{*} \in A_{v}$ and $g^{*} \in A_{w}$ for (known) $v$ and $w$ in $V$, then $\left\|\left(f^{*}\right)^{l}\right\|=0$ for every integer l. Find a reduced sequence $u$ representing $x g x^{-1}$; if $\|u\|>0$ then $g \notin F$. If $\|u\|=0$ then $x g x^{-1} \in A_{v}$, and $g \in F$ if and only if $x g x^{-1} \in\left\langle f^{*}\right\rangle \subseteq A_{v}$.

Corollary 4.2. Suppose $G$ satisfies the conditions of 3.6 and also

(i) the order problem is uniformly solvable for $A_{v}$ for every $v$ in $D$, and

(ii) the extended word problem for infinite cyclic subgroups is uniformly solvable for $A_{v}$, for every $v$ in $D$.

Then the extended word problem for the subgroup $\langle h\rangle$ is solvable for any loop $h$ in $G$ (or $\left.G_{0}\right)$.

Proof. By 3.8 the order of $h$ can be determined. If this is finite, equal to $n$, then any loop $g$ at $1_{\lambda h}$ is in $\langle h\rangle$ if and only if $g=h^{m}$ for some $1<m<n$, which is decidable by 3.6. If $h$ has infinite order, 4.1 applies.

So far, discussion has been of results of the kind "if decision problem $P$ is solvable for the vertex groups of a graph product $G_{0}$ then $P$ is solvable for $G_{0}$ ". To close, a class of graph products is determined for which the word problem and extended word problem for infinite cyclic subgroups are solvable.

The word problem and extended word problem for infinite cyclic subgroups (if they exist) are solvable for finite groups, countably generated free groups, countably generated free abelian groups (and countably generated free nilpotent groups), 
one-relator groups (see [7]) and sixth-groups (see [4]). Therefore, any of these groups (presented recursively) may be used in the initial stage of the construction described below.

DEFINITION 4.3. Let $\mathscr{D}$ be the class of connected directed recursive graphs. Let $\mathbb{Q}$ be a class of recursively presented groups which admit solutions to their word problems and to their extended word problems with respect to infinite cyclic subgroups. The class $\mathscr{D}(\mathscr{Q})$ consists of the groups which are presented as graph products of mapping cylinders $m(D, A)$ where

(i) $D$ is in $\mathscr{D}$,

(ii) $(D, A)$ is recursively presented,

(iii) for each $e$ in $D, A_{\lambda e}$ is an infinite cyclic or trivial group,

(iv) for each $v$ in $D$ such that $v \neq \lambda e$ for any $e$ in $D, A_{v} \in \mathbb{Q}$, and

(v) solutions to the word problem and the extended word problem with respect to infinite cyclic subgroups of $A_{v}$ are given uniformly from $V$.

LEMMA 4.4. If $G \in \mathscr{D}(\mathbb{Q})$ then the word problem and extended word problem for infinite cyclic subgroups of $G$ are solvable.

Proof. As $A_{e}\left(A_{\lambda_{e}}\right)$ is known to have a uniformly solvable extended word problem in $A_{\rho e}$ for every $e$ in $D$, the conditions of 3.6 and 4.1 are satisfied.

Now define $\mathcal{G}(\mathcal{Q})$ inductively by

$$
\mathcal{G}_{0}(\mathbb{Q})=\mathbb{Q}, \quad \mathcal{G}_{n}(\mathbb{Q})=\mathscr{D}\left(\mathcal{G}_{n-1}(\mathbb{Q})\right) \quad \forall n>1,
$$

and

$$
\mathcal{G}(\mathbb{Q})=\bigcup_{n} \mathcal{G}_{n}(\mathbb{Q}) \text {. }
$$

Trivially $\mathcal{G}_{n-1}(\mathscr{Q}) \subseteq \mathcal{G}_{n}(\mathbb{Q})$ since for any $G$ in $\mathcal{G}_{n-1}(\mathbb{Q})$ the group diagram $(D, A)$ with $D$ the trivial graph $D_{0}$ and $A_{0}=G$, has mapping cylinder $m(D, A)=G$. The final result follows immediately by induction, and extends the known results for simpler graph products (such as the generalised free products of [4, Theorem 5]) to the general case.

LEMMA 4.5. If $G \in \mathcal{G}(\mathcal{Q})$ then the word problem and extended word problem for infinite cyclic subgroups of $G$ are solvable.

\section{REFERENCES}

1. R. H. Crowell and N. Smythe, The subgroup theorem for amalgamated free products, HNN-constructions and colimits, Proc. Second Internat. Conf. Theory of Groups, Canberra, 1973, Lecture Notes in Math., vol. 372, Springer-Verlag, Berlin, 1974, pp. 241-280.

2. P. J. Higgins, The fundamental groupoid of a graph of groups, J. London Math. Soc. (2) 13 (1976), $145-149$.

3. Notes on categories and groupoids, Van Nostrand Reinhold Math. Studies, no. 32, Van Nostrand Rheinhold, London, 1971.

4. S. Lipschutz, An extension of Greendlinger's results on the word problem, Proc. Amer. Math. Soc. 15 (1964), 37-43.

5. R. C. Lyndon and P. E. Schupp, Combinatorial group theory, Modern Surveys in Math., no. 89, Springer-Verlag, Berlin, 1977.

6. Yu. I. Manin, A course in mathematical logic, Graduate Texts in Math., no. 53, Springer-Verlag, New York, 1977. 
7. J. McCool, The power problem for groups with one defining relator, Proc. Amer. Math. Soc. 28 (1971), 427-430.

8. J.-P. Serre, Arbres, amalgames, $S L_{2}$, Astérisque 46, Société mathématique de France, Paris, 1977 ; English transl., Trees, (translated by J. C. Stillwell), Springer-Verlag, Berlin, Heidelberg and New York, 1980.

9. N. Smythe, An analogue of Grushko's theorem for mapping cylinder groups, Notices Amer. Math. Soc. 23 (1976), A-419.

School of Mathematical and Physical Sciences, Murdoch University, Murdoch, W. A. 6150, Australia

Current address: Department of Mathematics, Melbourne University, Parkville, Victoria 3052 , Australia 